\title{
Quantum defects in fluorescent carbon nanotubes for sensing and mechanistic studies
}

Alexander Spreinat ${ }^{1,2}$, Maria M. Dohmen ${ }^{2}$, Jan Lüttgens ${ }^{3}$, Niklas Herrmann ${ }^{1,2}$, Lars F. Klepzig ${ }^{4,5}$, Robert Nißler ${ }^{1,2}$, Sabrina Weber ${ }^{2}$, Florian A. Mann ${ }^{2}$, Jannika Lauth ${ }^{4,5}$, Sebastian Kruss ${ }^{1,2,6 *}$

${ }^{1}$ Physical Chemistry II, Bochum University, Germany

${ }^{2}$ Institute of Physical Chemistry, Georg-August University Göttingen, Germany

${ }^{3}$ Institute of Physical Chemistry, Heidelberg University, Germany

${ }^{4}$ Institute of Physical Chemistry and Electrochemistry, Leibniz University Hannover, Germany

${ }^{5}$ Cluster of Excellence PhoenixD (Photonics, Optics, and Engineering - Innovation Across

Disciplines), Hannover, Germany

${ }^{6}$ Fraunhofer Institute for Microelectronic Circuits and Systems, Germany

*Corresponding author: Sebastian Kruss (sebastian.kruss@rub.de) 


\section{Abstract}

Single wall carbon nanotubes (SWCNT) fluoresce in the near infrared (NIR) and have been assembled with biopolymers such as DNA to form highly sensitive molecular sensors. They change their fluorescence when they interact with analytes. Despite the progress in engineering of these sensors the underlying mechanisms are still not understood. Here, we identify processes and rate constants that explain the photophysical signal transduction by exploiting sp $\mathrm{s}^{3}$ quantum defects in the $\mathrm{sp}^{2}$ carbon lattice of SWCNTs. As a model system we use ssDNA coated $(6,5)-\mathrm{SWCNTs}$, which increase their NIR emission $\left(\mathrm{E}_{11}, 990 \mathrm{~nm}\right)$ up to $+250 \%$ in response to the important neurotransmitter dopamine. In contrast, SWCNTs coated with DNA but with a low number of $\mathrm{NO}_{2}$-Aryl $\mathrm{sp}^{3}$ quantum defects decrease both their $\mathrm{E}_{11}(-35 \%)$ and defect related $\mathrm{E}_{11}{ }^{*}$ emission ($50 \%$ ) at $1130 \mathrm{~nm}$. Consequently, the interaction with the analyte does not change the radiative exciton decay pathway alone. Furthermore, the fluorescence response of pristine SWCNTs increases with SWCNT length, suggesting that exciton diffusion is affected. The quantum yield of pristine $(6,5)-\mathrm{SWCNT}$ increases in response to the analyte from $0.6 \%$ to $1.3 \%$ and points to a change in non-radiative rate constants. These experimental results are explained by a Monte Carlo simulation of exciton diffusion, which supports a change of two non-radiative decay pathways together with an increase of exciton diffusion (3 rate constant model). The combination of such SWCNTs with defects and without defects enables the assembly of ratiometric sensors with opposing responses at different wavelengths. In summary, we demonstrate how perturbation of a system with quantum defects reveals the photophysical mechanism and reverses optical responses. 


\section{KEYWORDS}

Carbon nanotubes, near infrared fluorescence, biosensors, photophysics, quantum defects, quantum color centers

\section{Introduction}

Single wall carbon nanotubes (SWCNTs) are rolled-up sheets of graphene and display fluorescence in the near infrared (NIR) $)^{1,2}$. Their fluorescence depends on their structure described by the chiral index $(n, m)$ and falls into the tissue transparency window $(800 \mathrm{~nm}-1700 \mathrm{~nm})$, which is beneficial for many (biomedical) applications ${ }^{3-5}$. Photophysics of SWCNTs is best described by mobile excitons ${ }^{6-8}$. During the typical lifetime of the exciton, it travels along the SWCNT axis and is affected by the local (chemical and dielectric) environment. Therefore, the picture of an exciton diffusing similar to a random walk and exploring its environment is able to explain several aspects of SWCNT photophysics, for example the length dependence of the quantum yield ${ }^{9}$. These properties make SWCNTs ideal building blocks for biosensors and fluorescent labels ${ }^{3,4}$. The SWCNT itself is the carrier of the excitons but to create specific interactions with analytes, the surface chemistry on SWCNTs plays a crucial role. Many non-covalent approaches including adsorption of biopolymers e.g. DNA ${ }^{10-13}$, DNA-peptide conjugates ${ }^{14,15}$, lipids $^{16,17}$, nanobodies ${ }^{18}$ or proteins ${ }^{19,20}$ have been successfully used to engineer selective surface chemistry. These approaches enabled a range of sensors for important biomolecules such as reactive oxygen species ${ }^{15,21,22}$, metabolites $^{23}$, sugars ${ }^{24,25}$, neurotransmitters ${ }^{26,27}$, proteins $^{17,28}$ and lipids ${ }^{29}$. However, how the molecular interaction with a target molecule affects SWCNT photophysics and decay pathways is still not understood. 
Most studies have been conducted on SWCNTs non-covalently functionalized with specific ssDNA sequences. One example are SWCNTs that increase their fluorescence in response to the important neurotransmitter dopamine ${ }^{26,30,31}$. Oxidation or reduction of the SWCNT/DNA complex by the analyte could not explain the observed changes in fluorescence intensity, even though there is a correlation between strong sensor responses and negative redox potential ${ }^{31,32}$. Experiments with dye-labeled ssDNA furthermore suggest that ssDNA conformation changes when it interacts with the analyte ${ }^{26}$. Additional evidence for such an interaction was found in molecular dynamics simulations, which showed that the phosphate group of the DNA sequence is pulled by the dopamine molecule towards the SWCNT surface ${ }^{33}$. Interestingly, DNA-sequences, which impart smaller fluorescence responses give rise to higher SWCNT starting fluorescence intensity. These findings suggest that the fluorescence increase is caused by dequenching 33,34 . The dissociation constants $\left(K_{\mathrm{D}}\right)$ for sensors assembled with different ssDNA sequences differ by several orders of magnitude, which indicates that the DNA sequence and most likely its conformation on the SWCNTs is of central importance ${ }^{30}$. Therefore, the organic phase around the SWCNT has a very strong influence on the fluorescence response but it is rather the structure and not the total number of adsorbed DNA molecules ${ }^{34}$. The mechanism might also depend on the exact surface chemistry because there are also designs for other molecules that yield sensors with fluorescence decrease and shifts in the spectrum ${ }^{35,36,21,37}$.

Despite the progress to engineer SWCNT-based sensors it is not understood how the interaction with the analyte changes exciton decay. A detailed investigation of the changes in exciton states and population is challenging because not all states are experimentally accessible ${ }^{38,39}$. One way to perturb exciton diffusion is to introduce $\mathrm{sp}^{3}$-hybridized carbon atoms (quantum defects) in the $\mathrm{sp}^{2}$ lattice of the SWCNT. It was long believed that defects in general quench NIR fluorescence ${ }^{32,40,41}$. 
However, reactions with $\mathrm{O}_{3}$ at very low levels lead to defects that create a new red-shifted fluorescence feature $\left(\mathrm{E}_{11}{ }^{*}\right)^{42}$. Reactions with diazonium salts cause also red-shifted emission features and even increase the quantum yield ${ }^{43,44}$. Such quantum defects or quantum color centers are useful to create new photophysical properties such as single-photon generation but are also useful for covalent functionalization ${ }^{45-47}$.

Here, we explore the effect of quantum defects in SWCNTs on chemical sensing by NIR fluorescent SWCNTs and develop a mechanistic photophysical model. More specifically, we explore which rate constants of exciton dynamics change during sensing. We hypothesized that $\mathrm{sp}^{3}$ quantum defects in the $\mathrm{sp}^{2}$ carbon lattice perturb exciton dynamics and sensing response and help narrow down the photophysical processes. Additionally, we study the impact of SWCNT length to probe the effect of diffusion and defect density and compare it with Monte-Carlo simulations of exciton diffusion. 


\section{Results and Discussion}

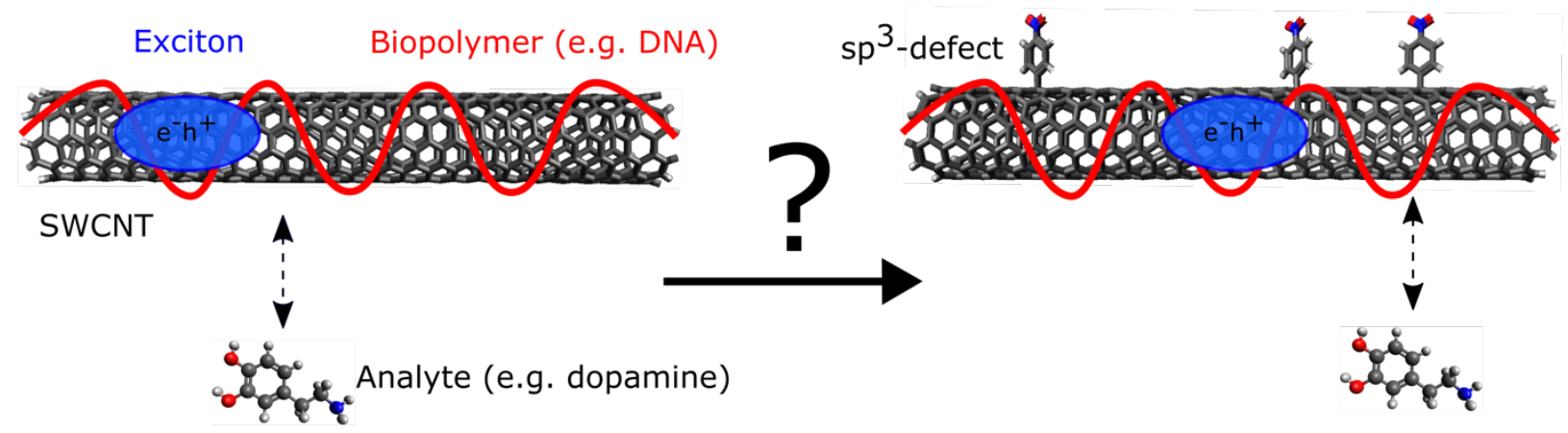

Figure 1: Effect of quantum defects on fluorescent sensing with SWCNTs. SWCNTs functionalized with biopolymers such as DNA can change their fluorescence when interacting with analyte molecules. One example are $(G T)_{10}-S S D N A$ functionalized SWCNTs, which increase their $\left(E_{11}\right)$ emission in response to the neurotransmitter dopamine. It is not known, how this interaction affects the diffusive walk of the exciton along the SWCNT, population of dark states and thereby exciton decay rate constants. $\mathrm{sp}^{3}$ defects in the carbon lattice ( $\mathrm{NO}_{2}$-Aryl) create a new optical decay pathway $\left(E_{11}{ }^{*}\right)$, which perturbs the optical transitions and is used to shed light on the mechanism and modify sensing.

To understand the mechanism of SWCNT-based sensors we designed experiments that perturb the possible exciton decay pathways and allow us to narrow down the involved processes (Figure 1). For most experiments, (GT) ${ }_{10}$-functionalized pristine CoMoCat-(6,5)-SWCNTs (from now on referred to as pSWCNTs) were used. Further purification ${ }^{48}$ was used for experiments in which spectral congestion by other chiralities could cause a bias (see materials and methods ${ }^{49}$ ).

Defects $\left(\mathrm{NO}_{2}\right.$-Aryl) were incorporated into $(6,5)-\mathrm{SWCNTs}$ by a reaction with diazonium salts (see materials and methods for details). In short, the quantum defects were introduced in SDBS suspended pSWCNTs and then the surfactant was exchanged to (GT) $)_{10}$-ssDNA. These SWCNTs with quantum defects are now referred to as $(\mathrm{GT})_{10}$-dSWCNTs. The new optical transition in dSWCNTs lies energetically below the dark states in $(\mathrm{GT})_{10}$-pSWCNTs ${ }^{44}$. One expects a defect 
density on the order of 1 defect per $25 \mathrm{~nm} \mathrm{SWCNT} \mathrm{length}{ }^{44}$. Additionally, it is known that one $(\mathrm{GT})_{10}$ molecule occupies around $2 \mathrm{~nm}$ of SWCNT length ${ }^{34}$. Consequently, the overall structure of the adsorbed DNA should not be changed much by the presence of the defects and $(\mathrm{GT})_{10^{-}}$ pSWCNTs and $(\mathrm{GT})_{10}$-dSWCNTs should be very similar besides the low number of quantum defects. The dopamine response for pSWCNTs and dSWCNTs was then compared for different concentrations of dopamine (Figure 2). As previously described ${ }^{26,30}$, dopamine increased the fluorescence of pSWCNTs in the experiments (up to +250\%). Surprisingly, the same SWCNTs with a low number of quantum defects (dSWCNTs) responded by a fluorescence decrease both for the $\mathrm{E}_{11}(-30 \%)$ and $\mathrm{E}_{11}{ }^{*}(-50 \%)$ transition $\left(100 \mu \mathrm{M}\right.$ dopamine). It is important to stress that the $\mathrm{E}_{11}$ peak results from excitons, which are freely diffusing along the SWCNT.
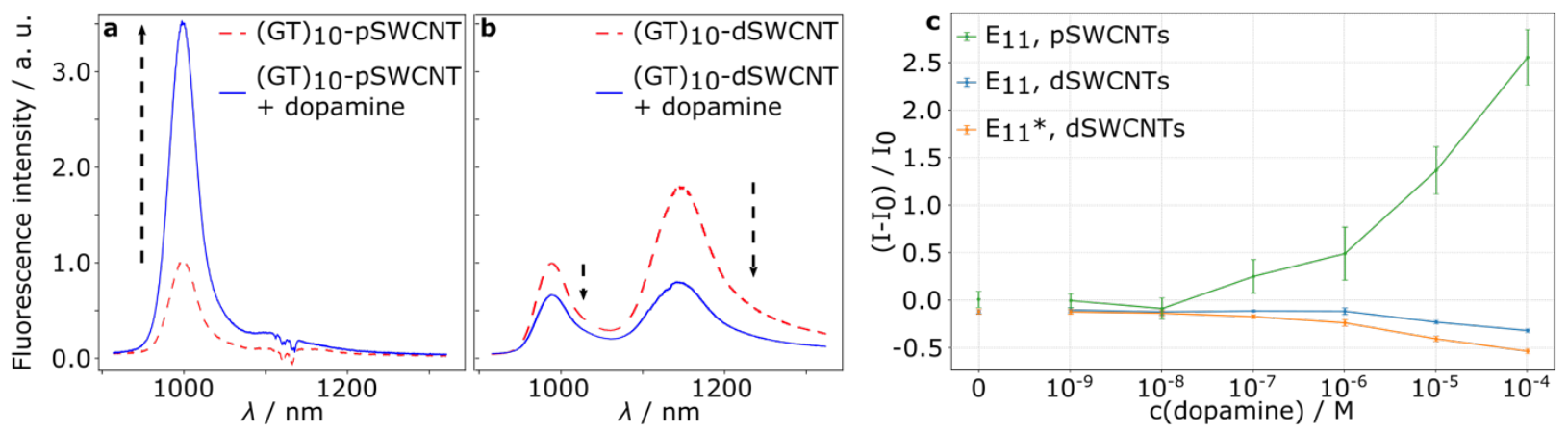

Figure 2: Quantum defects change the fluorescence response of carbon nanotubes to analytes. a NIR fluorescence spectrum $\left(E_{11}\right)$ before and after addition of dopamine $(100 \mu M)$ to $(G T)_{10}-p S W C N T s$. $\boldsymbol{b}$ Same for $(G T)_{10^{-}} d S W C N T s$ with a low number of $s p^{3}$ quantum defects (dSWCNTs). Spectra are normalized to the $E_{11}$ peak. $E_{11}$ and $E_{11}{ }^{*}$ changes for different analyte concentrations (1 $\mathrm{nM}-100 \mu \mathrm{M})$ for pristine and dSWCNTs. pSWCNTs increase their fluorescence with increasing dopamine concentration. In contrast, fluorescence of $d S W C N T$ s decreases for the $E_{11}{ }^{*}$ transition $(\approx-$ $50 \%)$ and to a smaller extent for the $E_{11}$ transition $(-\approx 30 \%)$. Error bars $=$ standard deviation. $n=3$. 
In contrast, the $\mathrm{E}_{11}{ }^{*}$ fluorescence signal results from excitons, which are trapped at the $\mathrm{sp}^{3}$-defect. Qualitatively, one can easily predict that the relative $\mathrm{E}_{11}$ signal of both dSWCNTs and pSWCNTs should increase if dopamine increases only the radiative rate constant, but this is not the case for pSWCNTs. Similarly, the relative pSWCNT $\mathrm{E}_{11}$ signal should increase and the $\mathrm{E}_{11}{ }^{*}$ signal of dSWCNTs should increase if the non-radiative rate constant(s) decreases by dopamine, but this is not the case for pSWCNTs. Therefore, the experimental results suggest a mechanism that involves multiple processes affected by the analyte.

The redistribution between the dark state and the bright exciton state depends on SWCNT length ${ }^{50}$ and consequently we assessed the dopamine response for SWCNTs of different length. The SWCNT where shortened via tip sonication and characterized by atomic force microscopy (AFM) (Supplementary Figure S1). Longer SWCNTs showed a stronger relative increase in the fluorescence intensity compared to shorter SWCNTs (Figure 3). This effect was observed for small concentrations of dopamine $(1 \mu \mathrm{M})$ and higher ones $(100 \mu \mathrm{M})$ (Supplementary Figure S2). The increase of fluorescence intensity of long SWCNTs for the sensing was up to three times larger than for short SWCNTs. These results indicate that dopamine influences the diffusion of the exciton (to quenching sites) because otherwise one would not expect an effect on the relative fluorescence changes (normalized to the starting intensity for short SWCNT). Tip sonication might introduce additional defects as direct quenching sites into the SWCNT. However, one would only expect an effect on the relative sensing response of these additional defects if they 'blocked' an exciton from "seeing" analyte molecules. The same concentration dependence for SWCNTs of different lengths indicates that the impact of undesired defects on the mechanism should be negligible (Figure S2). 

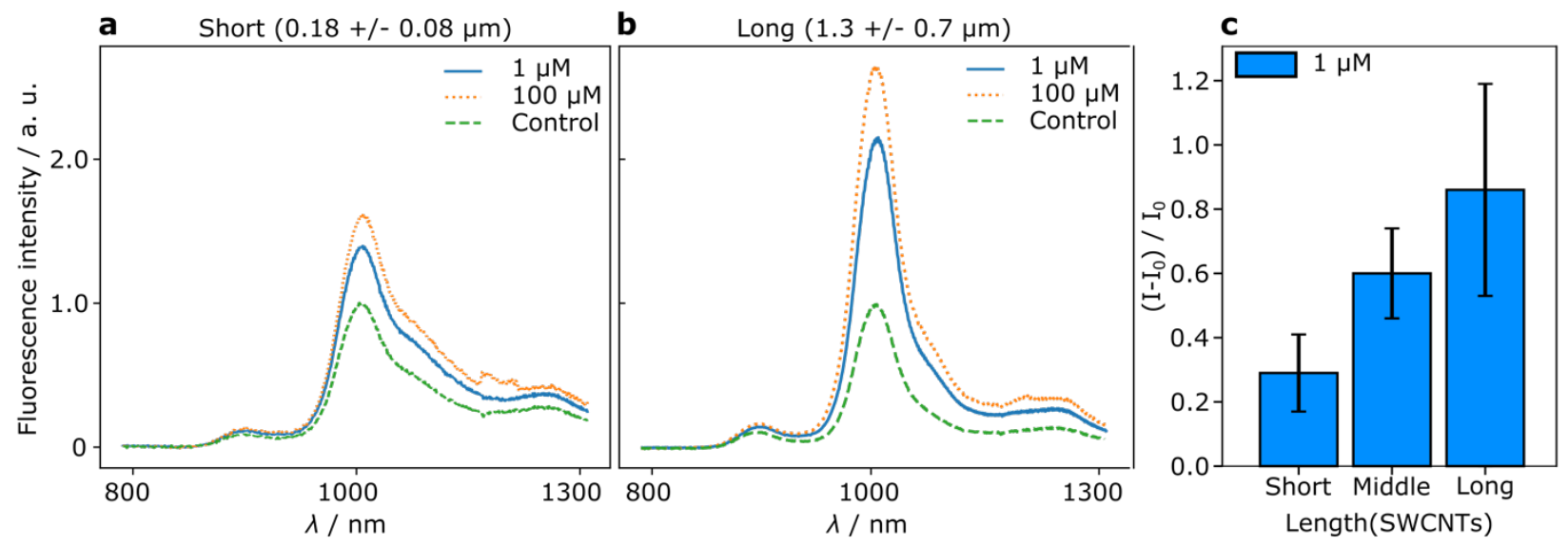

Figure 3: Effect of SWCNT-length on sensor response. a,b, Exemplary NIR fluorescence response of short (a) and

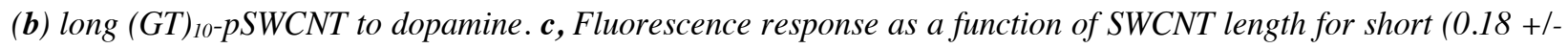
$0.06 \mu \mathrm{m})$, middle $(0.53+/-0.12 \mu \mathrm{m})$ and long $(1.3+/-0.7 \mu \mathrm{m}) \operatorname{SWCNTs}(n=3$, errors $=S D)$. Spectra are normalized to the starting intensity of the "short" SWCNTs.

Excitons could also dissipate by coupling to energetically similar vibrations of the solvent. This phenomenon is called electronic-to-vibrational energy transfer $(\mathrm{EVET})^{51}$. Using this pathway excitons could decay in a non-radiative way that is affected by the analyte. To test if this pathway changes during the interaction with the analyte, the dopamine response was measured in the two solvents $\mathrm{H}_{2} \mathrm{O}$ and $\mathrm{D}_{2} \mathrm{O} . \mathrm{H}_{2} \mathrm{O}$ has absorption bands in the NIR fluorescence window of the SWCNTs but $\mathrm{D}_{2} \mathrm{O}$ does not ${ }^{51}$. Therefore, different fluorescence responses were expected if dopamine affected solvation-related EVET. However, a significant difference in the dopamine response was not observed for the two solvents (Figure S3). Therefore, we conclude that direct EVET to the solvent is not a main factor. Additionally, the temperature dependence of the dopamine response was measured to test if thermal detrapping ${ }^{52}$ plays a role. Temperatures in a range that does not affect colloidal stability were selected $\left(5^{\circ} \mathrm{C}, 23^{\circ} \mathrm{C}\right.$ (room temperature, RT) and $50{ }^{\circ} \mathrm{C}$, Figure S4). The increases in fluorescence were similar, which rules out that the thermal trap depth of the defect state is modified by the analyte. So far, we only investigated processes that affect the fate of the 
exciton. An increase in absorption could also explain the analyte related fluorescence increase. However, the absorption cross section of the SWCNTs stayed constant during all experiments (Figure S5). It furthermore indicates, that not the radiative rate constant is changing during sensing as the absorption cross section is coupled via the oscillator strength to the radiative lifetime ${ }^{53}$.

These observations already rule out mechanisms in which the radiative decay from the $\mathrm{E}_{11}$ state is the single pathway affected by the analyte. To interpret the data more quantitatively, we modelled the processes after the creation of the exciton. For this purpose, we used a Monte-Carlo simulation that randomly creates excitons, lets them diffuse along the SWCNT axis while the rate constants determine their (stochastic) fate (Figure 4). We assumed two states, a bright and a dark state for the $\mathrm{E}_{11}$-exciton and a bright state for the $\mathrm{E}_{11}{ }^{*}$-defect state. Within our model and assumptions, we summarize the quenching at quenching $\operatorname{sides}^{9}$, endquenching and the rather unlikely multi-phonon decay $^{54}$ for the mobile $\mathrm{E}_{11}$ exciton to $k_{\mathrm{nr}}$ as the non-radiative decay and for the $\mathrm{E}_{11}{ }^{*}$ defect state multi-phonon decay and other possible non-radiative pathways ${ }^{51}$ to $k_{\mathrm{nr}}{ }^{*}$. Furthermore, we assumed that the defect states do not substantially change the non-affected transitions of dSWCNTs, given the low defect density. Based on the results above, we did not include an EVET pathway. Studies suggest that the intrinsic lifetime of the dark state is so long that the effective lifetime is only determined by endquenching ${ }^{50}$. Additionally, the bright states can also decay radiatively $\left(k_{\mathrm{r}}\right)$. Furthermore, the bright and dark state of the $\mathrm{E}_{11}$ exciton undergo an exchange of the population $\left(k_{\text {ic }}\right)$. Technically, by introducing defects into SWCNTs a bright state for the $\mathrm{E}_{11}{ }^{*}$-transition and a corresponding dark state arise ${ }^{39}$. However, both the dark and the bright state of the $\mathrm{E}_{11}{ }^{*}$ transition were summarized in a single state which can decay radiatively $\left(k_{\mathrm{r}}^{*}\right)$ or non-radiatively $\left(k_{\mathrm{nr}}{ }^{*}\right)$. The transition from the bright $\mathrm{E}_{11}$ state to the $\mathrm{E}_{11}{ }^{*}$ state is purely diffusion driven, as it is assumed in general for the trapping of excitons at $\mathrm{sp}^{3}$-defects ${ }^{55}$. We assumed both $\mathrm{E}_{11}$ states to be mobile, with 
the dark state having around one third of the diffusion constant of the bright exciton ${ }^{55,56}$. A 'detrapping' from the defect side back to the mobile exciton is also considered. Some previous studies assume that the exciton is almost immediately quenched after detrapping ${ }^{52}$. However, in our simulation a return to the mobile state leads to quantum yields closer to the measured values. A schematic of the states and transitions are visualized in Figure 4 a and b. In general, the decay pathways of the $\mathrm{E}_{11}{ }^{*}$-states are summed up in our model to the same simplified steps as for the $\mathrm{E}_{11^{-}}$ states, but the physics behind them differ strongly. As the $\mathrm{E}_{11}{ }^{*}$-states are localized at the defect end quenching 9 and quenching at other quenching sides along the SWCNTs cannot happen. The relaxation pathways of these states therefore follow other physics and are influenced by other processes and can be treated completely separately from the behavior of the transitions of the $\mathrm{E}_{11^{-}}$ states.

To perform a realistic simulation, boundary conditions are necessary. Therefore, the absolute photoluminescence quantum yield (PLQY) with and without dopamine was measured (Table 1, Table S1, Table S2, Figure S6). Used rate constants are found in various literature sources ${ }^{9,55}$ and are summarized in Table S3. Rate constants that are available neither in literature nor directly accessible in experiments were iterated to the point where the general quantum yield resulting from the simulation was in good agreement with the measured PLQY. Additionally, a dataset of femtosecond transient absorption measurements was collected for the dopamine sensing for samples of the pSWCNTs. Here, no change on the time scale up to $10 \mathrm{ps}$ for the general depopulation of the mobile exciton was observed (see Figure S7), which indicates for the expected changes to be on longer time scales, which also was used as an indirect boundary condition for the simulation. 
Table 1: PLQY measured for the samples.

\begin{tabular}{|l|l|l|}
\hline & $\begin{array}{l}(\mathrm{GT})_{10}-\mathrm{pSWCNT} \\
\text { purified }\end{array}$ & $\begin{array}{l}(\mathrm{GT})_{10} \text {-dSWCNT } \\
\mathrm{NO}_{2} \text {-Aryl-defect } \\
\left(\mathrm{E}_{11}, \mathrm{E}_{11}{ }^{*}\right)\end{array}$ \\
\hline PLQY & $0.64+/-0.05 \%$ & $\begin{array}{l}0.85+/-0.09 \% \\
(0.22 \%, 0.63 \%)\end{array}$ \\
\hline $\begin{array}{l}\text { PLQY } \\
\text { (with } 100 \mu \mathrm{M} \\
\text { dopamine) }\end{array}$ & $1.3 \%$ & $0.54 \%$ \\
\hline
\end{tabular}

Based on the experimental data, the following scenarios could explain the fluorescence changes in pSWCNTs induced by the analyte: 1 . The radiative decay rate increases, 2 . the non-radiative decay rate decrease or 3. the transition rate from the dark state to the bright state increases, either by a change in $k_{\text {ic }}$ populating the bright state or by an increase of the lifetime of the dark state. In the latter case, an influence of SWCNT length seems likely because end quenching dominates the dark state's life time ${ }^{50}$. All these scenarios explain the behavior of the pSWCNTs but they cannot explain the fluorescence changes of dSWCNTs (Supplementary Table S4) and imply that that there is more than one process affected by the analyte. The length dependence of the sensing response (Figure 3) also suggests that end quenching of the SWCNTs plays a role. It could be explained either by a different lifetime of the exciton (dark and/or bright) or due to an increased exciton diffusion coefficient. We also varied the defect density (ratio of $\mathrm{E}_{11}$ and $\mathrm{E}_{11}{ }^{*}$ peak as proxy). Interestingly, the dopamine response for dSWCNTs was independent of defect density (Figure S8). Previous studies showed a defect density of 1 defect per $25 \mathrm{~nm} \mathrm{SWCNT}$ and we expect that 
our densities are in a similar range ${ }^{44}$. This result suggests that the overall situation for a free or trapped exciton that is affected by dopamine does not depend on how far away the next defect is.

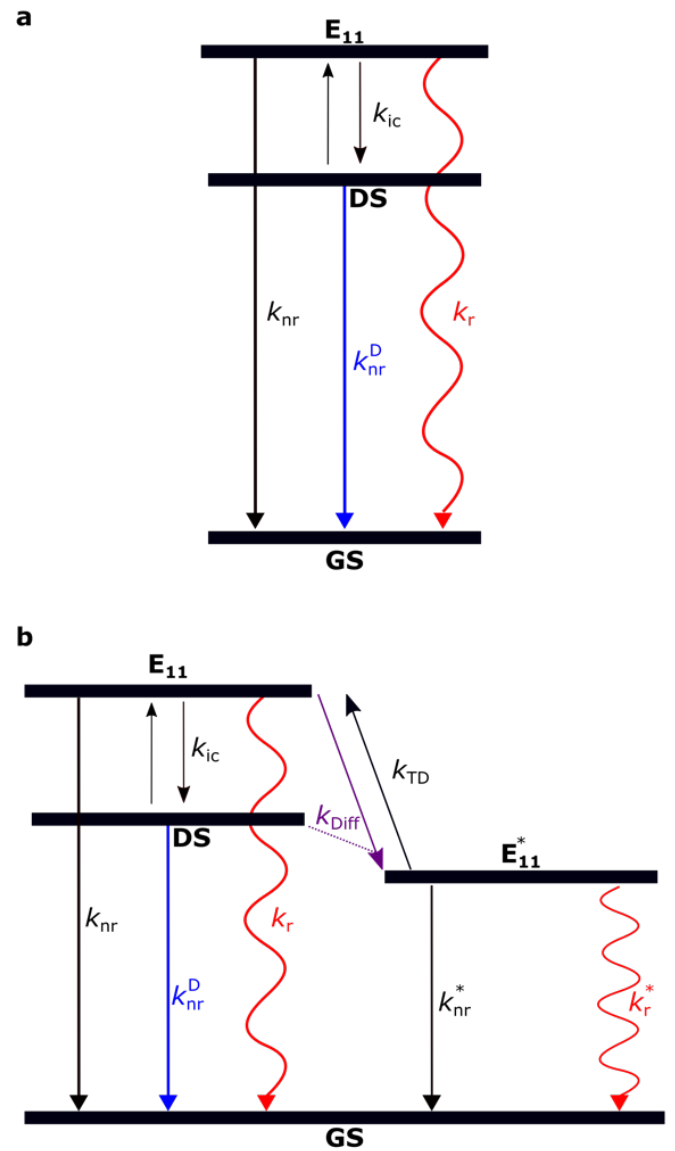

c

1) Initate SWCNT of length $L$

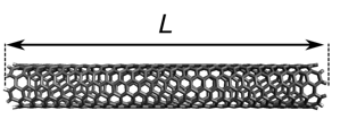

2) $n$ randomly placed defects on SWCNT

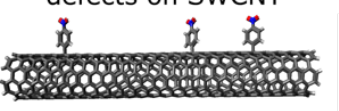

3) Initiate mobile exciton

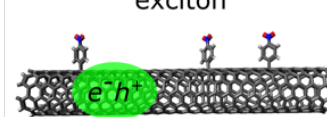

$k_{r}$ : Radiative decay

$k_{\mathrm{n} r}$ : Non-radiative decay

$k_{i c}$ : Interconversion between

excited and dark state

$k_{\text {Diff: }}$ : Trapping of exciton if it hits a defect site along the simulated diffusion path $k_{\mathrm{TD}}$ : Thermal detrapping $t_{j}$ : Time step $j$

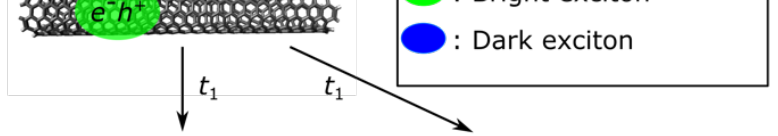

4a) Free diffusion in excited state

4b) Free diffusion in dark state

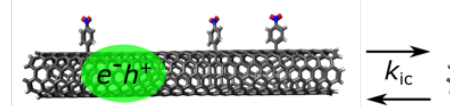

i t

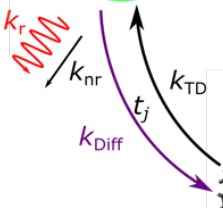

4c) Trapped exciton

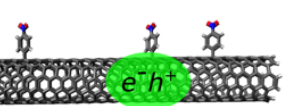

$k_{\mathrm{r}}^{*} \sum \mid k_{\mathrm{nr}}^{*}$

Figure 4: Monte-Carlo model of exciton diffusion and decay pathways altered by the analyte. a Energetic states (simplified) and transitions for pristine SWCNTs ( $p S W C N T)$ that could be involved in analyte sensing. $E_{11}$ indicates the bright state, DS is the dark state and GS is the ground state. $\boldsymbol{b}$ Similar schematic for SWCNTs with defects (dSWCNTs). The nomenclature for the states remains, while the analogous defect states are marked with "*”. Additionally, diffusion enables trapping in defect states and detrapping. $\mathrm{c}$ Simulation concept: A SWCNT of a certain length L (e.g., $750 \mathrm{~nm})$ is created with/without a certain number of randomly distributed defects (e.g. one defect per $30 \mathrm{~nm})$. The diffusing exciton can decay radiatively $\left(k_{r}\right)$ or non-radiatively $\left(k_{n r}\right)$ to the ground state $(G S)$, it can transition to a dark state (DS), it can be trapped at a defect side or quenched at the end of the SWCNT. Trapped excitons can decay via a radiative $\left(k_{r}{ }^{*}\right)$, a non-radiative $\left(k_{n r}{ }^{*}\right)$ path or thermal detrapping. The used (starting) rate constants are summarized in Table S3. 
The simulation allowed us to test different scenarios and rate constants in silico under all these experimental conditions. A summary of the individual simulation steps is shown in Figure 4c. The schematic shows only single $\mathrm{NO}_{2}$-Aryl-functionalization for a better visibility but in reality $\mathrm{sp}^{3}$ defects appear as "ortho" and "para" position defects ${ }^{57}$. Similar photophysical simulations have shown to lead to fruitful results but have not been used to explore sensing by SWCNTs ${ }^{58}$. Based on the experimental data, it is already possible to rule out a couple of mechanisms by purely qualitative considerations as discussed above. However, the simulation allowed us to change multiple rate constants at the same time and observe the effect. We found that the experimental results are best supported by a model (Figure 5) in which 3 parameters change at the same time (3RC model). In this model $k_{\mathrm{nr}}$ decreases, $k_{\mathrm{nr}}{ }^{*}$ and the the exciton diffusion constant $\left(D_{\mathrm{e}}\right)$ increases by the analyte.
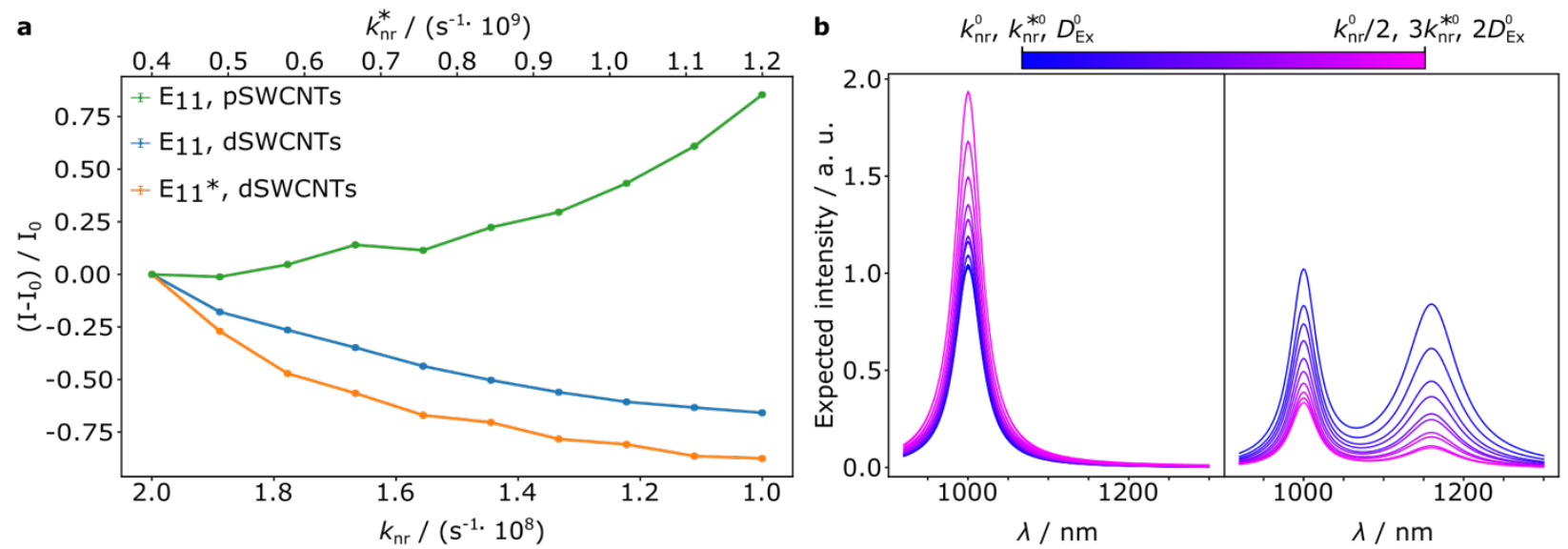

Figure 5: Simulated fluorescence response for the three-rate constant (3RC) model. The Monte-Carlo simulation was tested for different scenarios. Here, a simulation is shown (three rate constant model, $3 R C$ model) in which $k_{n r}$ decreases and $k_{n r}{ }^{*}$ as well as the exciton diffusion constant $\left(D_{e}\right)$ increase in response to the analyte. a shows the expected intensity changes of $d S W C N T$ s and $P S W C N T$ s when changing the constants (corresponding to the analyte concentration) $k_{n r}{ }^{*}$ (up to $\left.3 x\right), k_{n r}(1 / 2 x)$ and $D_{e}(2 x)$ of the initial value. $\boldsymbol{b}$ shows the corresponding simulated spectra for pSWCNTs and dSWCNTs (with defects) matching the experimental results in figure 2. 
It is known that that the diffusion coefficient of the exciton depends on the dielectric environment and therefore it is likely that exciton diffusion is affected by the presence and binding of the analyte $^{59}$. In this picture, the analyte causes conformational changes of the adsorbed DNA, which changes the potential landscape through which the exciton diffuses. A necessary additional explanation is that in addition to the changes in the exciton diffusion mentioned above, $k_{\mathrm{nr}}{ }^{*}$ is increasing during the sensing event while $k_{\mathrm{nr}}$ is decreasing. This also explains the phenomenological explanations from previous studies ${ }^{33}$, which basically assume that an exciton that hits a DNA strain upon the SWCNT surface is quenched, therefore $k_{\mathrm{nr}}$ would be increased for the sensing in pSWCNTs. The established hypothesis for the molecular interaction between the dopamine molecules and the DNA assumes that the phosphate backbone interacts with the vicinale OH-groups of the dopamine molecule and that therefore the DNA moves closer to the SWCNT surface and quenching of the excitons is reduced ${ }^{33}$. Other simulated scenarios could qualitatively not reproduce the results and therefore we suggest the 3RC-model as the mechanism for sensing. Based on the measurements and simulations carried out, the fluorescence responses for two other important analytes and SWCNT-based sensors (ascorbic acid, riboflavin) were tested ${ }^{31,35}$. For riboflavin, a strong decrease in fluorescence intensity was observed for pSWCNTs. For dSWCNTs, we observed only a very small decrease of both $\mathrm{E}_{11}$ and $\mathrm{E}_{11}{ }^{*}$ (Figure S10). This data can be interpreted in such a way that the riboflavin interaction directly quenches excitons or let excitons go into the dark state of pSWCNTs. In dSWCNTs the new defect state serves as an exciton trap and is a competing process. Therefore, the quenching effect of riboflavin is nearly completely abolished. For ascorbic acid, fluorescence changes for pSWCNTs and dSWCNTs are similar to dopamine sensing. The absolute changes in fluorescence can be explained by differences in the rate constants, but the general mechanism is most likely the same (Figures S10 and S11). 

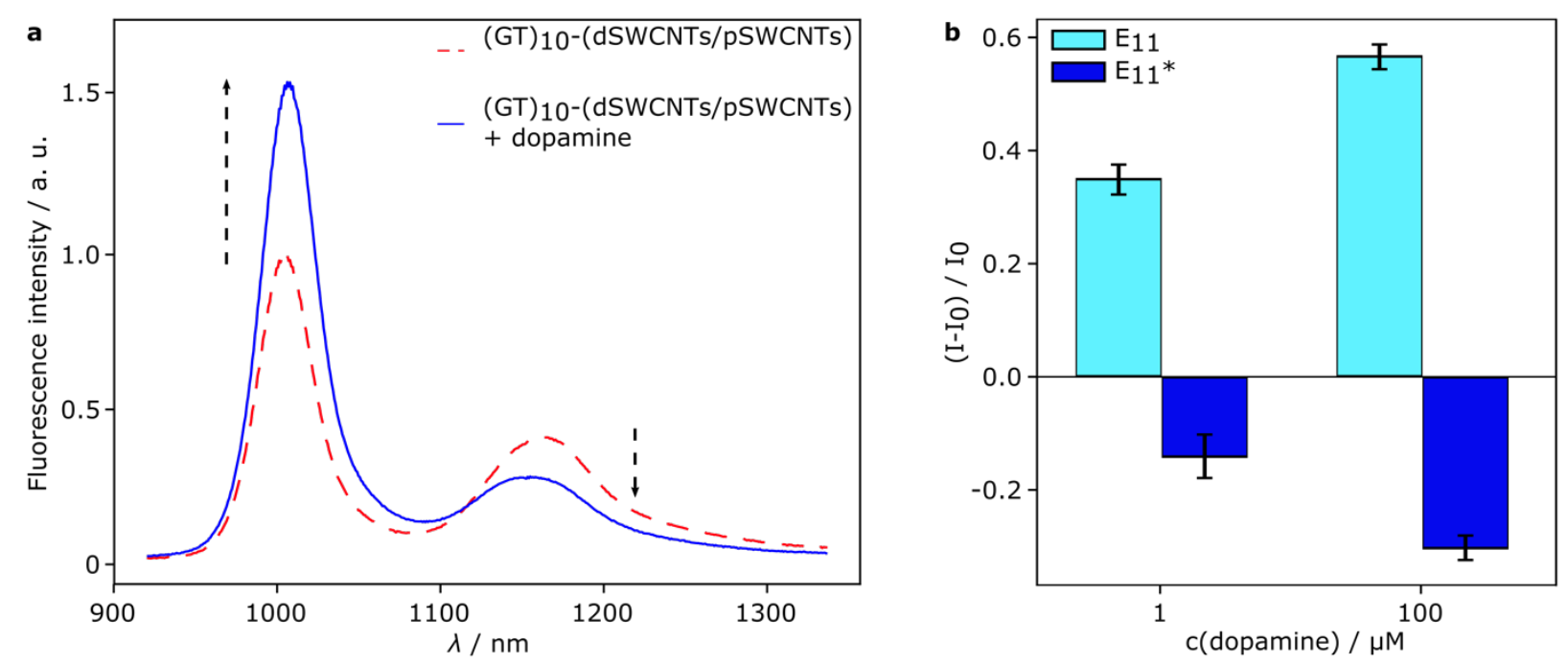

Figure 6: Ratiometric sensing of dopamine using quantum defects. a, Fluorescence spectrum of mixed (GT) 10 dSWCNTs/pSWCNTs before and after the addition of dopamine $(100 \mu \mathrm{M})$. The $\mathrm{E}_{11}$ emission increases $(+60 \%)$ and the $\mathrm{E}_{11}{ }^{*}$ transition decreases (-30\%), which enables ratiometric sensing at two different wavelengths. b Ratiometric fluorescence response to dopamine $(1 \mu \mathrm{M}, 100 \mu \mathrm{M}) \cdot \mathrm{n}=3$, error $=\mathrm{SD}$.

Our mechanistic insights provide a framework for understanding different SWCNT-based sensors. The exact surface chemistry might affect the absolute changes in rate constants but the approach to perturb the sensing response with quantum defects can be applied to all sensors. Narrowing down the involved rate constants can furthermore serve as a guideline to improve sensitivity by tailoring signal transduction.

Different fluorescence responses at different wavelengths opens opportunities for advanced sensing schemes. We used it to establish ratiometric sensing at two different wavelengths (Figure 6). Such approaches are desired in biosensing because they are more robust and promise higher signal $/$ noise ratios ${ }^{48}$. The dopamine response almost equals the average of the expected dopamine responses for two independent pSWCNT and dSWCNT samples, which further underlines that the presence of $\mathrm{NO}_{2}$-Aryl-units does not interfere with the overall DNA structure on the SWCNTs. Consequently, quantum defects are a useful tool to create spectrally encoded ratiometric sensors. 


\section{Discussion}

In this work, we placed quantum defects in SWCNTs to perturb the fluorescence response to analyte molecules. Other studies focused more on covalent functionalization chemistry ${ }^{60}$. The data provided mechanistic insights into the sensing mechanism of SWCNT-based fluorescent sensors. We could rule out that energy transfer to vibrations of the solvent playd a major role. Additionally, the length dependence highlights that exciton diffusion and/or end quenching is involved. The surprising finding that a small number of quantum defects completely reverses the fluorescence change led to the conclusion that multiple rate constants are affected by the analyte.

These data and the additional Monte-Carlo exciton diffusion simulation suggested that a model in which three rate constants are changed by the analyte is most likely (3RC-model). In this model a decrease of the $k_{n \mathrm{r}}$, an increase of the exciton diffusion constant $D_{\mathrm{e}}$ and an increase in the nonradiative decay $k_{\mathrm{nr}}{ }^{*}$ is caused by the analyte (dopamine). This model is in agreement with experimental results from previous studies and establishes a kinetic understanding of the sensing mechanism ${ }^{33}$. It allows also to understand other analytes as we show for riboflavin and ascorbic acid. For different surface chemistries analyte rate constants will most likely change in different ways but our work provides a framework for other analytes and how quantum defects can be used to mechanistically narrow down possible mechanisms. Such insights will also further guide the design of novel sensors with enhanced sensitivity and selectivity. Additionally, we could show that the combination of SWCNTs with defects and without defects enables ratiometric sensing approaches. One other major advantage of SWCNT-based sensors with quantum defects is the higher quantum yield compared to normal SWCNTs (Table 1). 
SWCNT-based sensors have recently been used as diagnostic tools that detect pathogens or identify disease markers ${ }^{23,61}$. Furthermore, they are used to reveal fundamental insights into chemical communication between cellular networks ${ }^{62}$ or as macroscopic imaging tool to study plant health ${ }^{15,63,64}$. These examples show the large potential of SWCNT-based NIR sensing and imaging. Our insights provide mechanistic guidelines to further tailor such sensors. Recently, it was also shown that it is possible to grow peptide chains on quantum defects ${ }^{46}$, which opens further possibilities to change the chemical environment for the trapped exciton and thereby decay pathways. Therefore, using different types of quantum defects both mechanistic insights and general advances in sensing are envisioned.

\section{Conclusion}

In summary, this work establishes $\mathrm{sp}^{3}$ quantum defects in the lattice of a nanomaterial as a tool to both reveal photophysical mechanisms and vary sensing responses. We use it to identify the most likely mechanism for SWCNT-based sensors and show that multiple rate constants are involved. Additionally, this work enables mechanism-guided chemical design of even brighter and more sensitive sensors. 


\section{Materials and Methods}

\section{Chemicals}

All chemicals, unless otherwise stated, were supplied from Sigma Aldrich. $\mathrm{D}_{2} \mathrm{O}$ had a deuterium share of $99.9 \%$. Milli- $\mathrm{Q}^{\mathrm{TM}}$-water was used for all experiments.

\section{Preparation of (GT) 10 functionalized single-walled carbon nanotubes (SWCNTs)}

A SWCNT stock suspension (2 mg (6,5)-chirality enriched SWCNT (ComoCat, Lot MKBZ1159V) per $1 \mathrm{~mL}$ water) was prepared. $100 \mu \mathrm{L}$ of this suspension were spun down (16100 $\mathrm{g}, 3 \mathrm{~min}$ ), the supernatant was discarded and $200 \mu \mathrm{L}$ of a $2 \mathrm{mg} \cdot \mathrm{mL}^{-1}$ solution of ssDNA (supplied by Sigma-Aldrich, desalted) in 1x PBS (phosphate buffered saline) (in $\mathrm{H}_{2} \mathrm{O}$ or $\mathrm{D}_{2} \mathrm{O}$, respectively) was added. The mixture was tip-sonicated (Fisher Scientific Modell 120 Sonic Dismembrator, 15 $\min , 40 \mathrm{~W}$, cooled in an ice-water bath) and subsequently centrifuged $\left(16100 \mathrm{~g}, 30 \mathrm{~min}, 5{ }^{\circ} \mathrm{C}\right)$ to remove remaining bundled nanotubes and metallic residues. The upper $90 \%$ of the resulting supernatant was used for further experiments.

\section{Preparation of sodium cholate single-walled carbon nanotubes (SWCNTs) with different}

\section{lengths}

For each sample, $600 \mu \mathrm{L}$ of the above mentioned SWCNT stock solution was spun down and the supernatant was discarded. $1.2 \mathrm{~mL}$ of a $10 \mathrm{mg} \cdot \mathrm{mL}^{-1}$ sodium cholate solution in water was added. The resulting suspensions were tip-sonicated for $7 \mathrm{~min}, 30 \mathrm{~min}$ or $4 \mathrm{~h}$, respectively (Fisher Scientific Modell 120 Sonic Dismembrator, 36, 48 or 60 watts respectively). The resulting dispersions were centrifuged $\left(16,100 \mathrm{~g}, 30 \mathrm{~min}, 21^{\circ} \mathrm{C}\right)$ to remove remaining bundled nanotubes and metallic residues. The upper $90 \%$ of the resulting supernatant was used for further 
experiments. For these experiments, $100 \mu \mathrm{L}$ of the SC-SWCNT dispersions $(c=32 \mathrm{nM})$ were mixed with $100 \mu \mathrm{L} 1 \times$ PBS and $100 \mu \mathrm{L}$ of a $2 \mathrm{mg} \cdot \mathrm{mL}^{-1}$ solution of $(\mathrm{GT})_{10}$. The mixture was dialysed in $1 \mathrm{kDa}$ MWCO dialysis bags (Spectra/Por, Spectrum Laboratories Inc.) against 1xPBS (4x $500 \mathrm{~mL}$ ). The resulting dispersions were centrifuged $\left(30 \mathrm{~min}, 16100 \mathrm{~g}, 21{ }^{\circ} \mathrm{C}\right.$ ) and the supernatant was used for further experiments.

\section{Atomic Force Microscopy}

The cholate-SWCNT dispersions were diluted by the factor 10 and $10 \mu \mathrm{L}$ of each sample were spin-coated (RT, $1000 \mathrm{rpm}$ ) on muscovite mica. The samples were rinsed with water and dried in a nitrogen stream. AFM measurements were performed using the intermittent contact mode (scanrate $=2 \mathrm{~Hz}$ or $1 \mathrm{~Hz}, 512$ lines) on an Asylum Research MFP-3D Infinity with rectangular cantilevers (Opus, MikroMasch Europe, Al-coated, tetrahedral-shaped tip, $f_{\text {res }}=300 \mathrm{kHz}, k=26$ $\mathrm{N} \cdot \mathrm{m}^{-1}$ ). Image analysis was done with Gwyddion ${ }^{65}$. The images were background-corrected using a 2nd grade polynome and the lowest value was set to zero.

\section{Determination of the SWCNT concentration}

The ssDNA-SWCNT dispersions' concentration was estimated using NIR absorption measurements as previously described ${ }^{34}$. The stock solutions were diluted to achieve a peak height at the $\mathrm{E}_{11}$-transition not exceeding an absorption of 1. The $\mathrm{E}_{11}$ absorption at approx. $991 \mathrm{~nm}$ was used for the calculation according to ${ }^{34}$. All dispersions were diluted to a concentration of $0.3 \mathrm{nM}$ using $1 x$ PBS (in $\mathrm{H}_{2} \mathrm{O}$ or $\mathrm{D}_{2} \mathrm{O}$, respectively) and stored at room temperature. 


\section{Fluorescence spectroscopy in the presence of dopamine}

The fluorescence measurements were performed in clear 96-well plates (F96 Maxisorb nuncimmuno, Thermo Fisher). A DPSS (Diode-pumped solid state) $561 \mathrm{~nm}$ continuous wave laser (Laserquantum gem 561) was used for excitation. The light was passed through an Olympus IX73 inverted microscope (10x Olympus UPlanFL N-FN26.5-0.30 10x objective). The SWCNT NIR emission was guided to a spectrometer (Shamrock 193i spectrograph) equipped with an Andor iDUS InGaAs 491 array NIR InGaAs detector. Per well, $180 \mu \mathrm{L}$ of the $0.3 \mathrm{nM}$ SWCNT dispersion was mixed with $20 \mu \mathrm{L}$ of a dopamine hydrochloride solution (in $1 \mathrm{x}$ PBS) with $c=1 \mathrm{mM}$ or $10 \mu \mathrm{M}$ (start concentration). For control samples, $20 \mu \mathrm{L} 1 \mathrm{x}$ PBS was added. The resulting mixtures were measured directly after preparation $(t<10 \mathrm{~min})$.

\section{Purification of SWCNTs}

The purification of $(6,5)-\mathrm{SWCNTs}$ was performed according to a previously reported aqueous two-phase extraction (ATPE) protocol from Li et al. ${ }^{49}$ Using a three step approach SWCNT chiralities were separated between two aqueous phases, containing dextran (MW 70000 Da, 4\% $\mathrm{m} / \mathrm{m}$ ) and PEG (MW $6000 \mathrm{Da}, 8 \% \mathrm{~m} / \mathrm{m}$ ) with varying $\mathrm{pH}$-values due to $\mathrm{HCl}$ addition. The final B3 (bottom)-phase yielded nearly monochiral $(6,5)-\mathrm{SWCNTs}$, which were diluted with DOC to obtain a stable 1\% DOC-SWCNT solution. Further dialysis with a $300 \mathrm{kDa}$ dialysis bag against $1 \%$ DOC removed the dextran polymer which was used for the SWCNT separation. In order to transform the purified SWCNTs into sensors again, a surface exchange had to be performed. For that $150 \mu$ of purified $(6,5)-S W C N T$ s in a solution of $1 \%$ DOC ( 2 absorption at $986 \mathrm{~nm}), 25 \mu 1$ of PEG (MW $6 \mathrm{kDa}, 25 \% \mathrm{~m} / \mathrm{v})$ and $30 \mu \mathrm{l}$ of conjugated (GT) ${ }_{10}$-DNA $(2.5 \mathrm{mg} / \mathrm{mL}$ in H2O) were mixed. After that, $270 \mu \mathrm{L}$ of methanol was added in three steps of $90 \mu \mathrm{L}$ in order to replace the 
DOC with DNA on the SWCNT surface. $600 \mu \mathrm{L}$ of iso-propyl alcohol was used to crush the SWCNT-DNA-complex out. The solution was centrifuged at $16100 \mathrm{~g}$ for $2 \mathrm{~min}$. Then, the supernatant was removed and the pellet was dried to remove last traces of other organic solvents. After one precipitation cycle, the nanotube pellet was directly redispersed in $200 \mu 11$ x PBS and characterized by absorption spectroscopy.

\section{Introduction of sp $^{3}$-defects into SWCNTs}

For the introduction of $\mathrm{sp}^{3}$-defects into SWCTNs, samples of $180 \mu \mathrm{L}$ of a previously purified $(6,5)-$ SWCNT-solution in 1\% SDBS with a concentration of $10 \mathrm{nM}$ were placed in wells of a 96-well plate (F96 Maxisorb nunc-immuno (Thermo Fisher)). $20 \mu \mathrm{L}$ of a freshly prepared 4-nitrophenyl diazonium tetrafluoroborate solution in water with a concentration of $100 \mu \mathrm{M}$ were added to each sample (final concentration in the well: $10 \mu \mathrm{M}$ ). After the addition, the wells were illuminated with a LumidoxTM (Analytical Sales \& Services, Inc, Flanders NJ, USA) 96 green LED array with an LED current of $25 \mathrm{~mA}$. The reaction was controlled and observed using fluorescence spectroscopy and the development of the $\mathrm{E}_{11}{ }^{*}$-Peak. After that, the reaction mixtures were transferred to spin filters (VivaspinTm 500, MWCO $=100 \mathrm{kDa}, \mathrm{V}=500 \mu \mathrm{L}$, Sartorius, Göttingen, Germany) and centrifuged at 12,000 g. The dSWCNTs, which precipitated on the membrane of the filter, were washed 4 times with $450 \mu \mathrm{L}$ of water. Between each washing step, the sample was centrifuged again. After that, the SWCNTs were resuspended in the desired surfactant, in this case DOC. For resuspension, the sample was tip-sonicated in an cooled ice bath ( $30 \%$ amplitude, $36 \mathrm{~W}, 5 \mathrm{~min})$. 


\section{Measurement of photoluminescence quantum yields}

The PL quantum yield (PLQY) of the dispersions was determined by an absolute approach measuring the ratio of emitted to absorbed photons, as reported previously ${ }^{66,67}$. Firstly, a cuvette containing the solvent and the respective PBS concentration of the sample was placed within an integrating sphere (LabSphere, Spectralon Coating) and the intensity of the excitation laser (561 $\mathrm{nm}$, WhiteLase SC400 supercontinuum laser, Fianium Ltd.) and as well as the dark counts of the PL emission region without SWCNTs were recorded. The signal from the sphere was transmitted via an optical fiber and coupled into an Acton SpectraPro SP2358 (grating 150 lines $/ \mathrm{mm}$ ) spectrometer with an OMA-V InGaAs line camera (Princeton Instruments) for spectra acquisition. Subsequently, the respective SWCNT sample was inserted and both the emission spectra an attenuated laser peak were measured. To account for wavelength dependent transmissivity and reflectivity of all optical components as well as the absorption of solvent and PBS, the emission of a broadband light source with stabilized color temperature (IR Light Source SLS202L/M, Thorlabs Inc.) was recorded for both spectral regions with a cuvette containing the solvent and the respective PBS concentration. A correction function was calculated by dividing these data by the black body spectrum of the lamp's color temperature. Finally, the collected spectra were multiplied by the correction function and the wavelength to ensure direct proportionality between detector counts and photons. The difference of the integrated laser intensities was taken as a measure for the absorbed photons and the difference of the integrated spectral region of the SWCNT emission was taken as a measure for the emitted photons. The ratio of both quantities gives the PLQY of the sample. Due to the low emitter concentration, self-absorption was neglected. The obtained PLQY can therefore be viewed as conservative. 


\section{Transient Absorption Spectroscopy Measurements}

Ultrafast broadband transient optical absorption spectroscopic measurements were performed on a set-up similar as described in literature ${ }^{68,69}$. Briefly, a 100 fs laser pulse train from a Ti:sapphire amplifier system (Spectra-Physics, Spitfire ACE) centered at $800 \mathrm{~nm}$ is used and split (90:10) to generate a pump and a probe beam. A broadband super continuum is generated by nonlinear processes inside $\mathrm{a} \mathrm{CaF}_{2}$ or sapphire crystal $(320-1600 \mathrm{~nm})$ with $10 \%$ of the fundamental. The arrival of the probe beam with respect to the pump is varied by using a delay stage with a range of up to $8 \mathrm{~ns}$. The pump beam energy is controlled by nonlinear frequency mixing in an optical parametric amplifier (OPA) system (TOPAS). The pump pulse is dumped after the photoexcitation of the sample, while the probe light is led to a fiber-coupled detector array (Ultrafast, Helios FIRE).

\section{Simulation Algorithm}

The simulation was written in Python to model the movement of an exciton on the SWCNT. For this purpose, the model is simplified to a 4-state model. In the addition to the ground state, there are a bright and a dark mobile $S_{11}$ state, which are in equilibrium. The bright state can decay both radiative and non-radiatively, while the dark state only allows for non-radiative decay. Both states can transition to a radiative state $S_{11}$ * located at the defect site. Thermal detrapping into $S_{11}$, radiative and non-radiative decay are the depopulating channels for the defect state. The movement of the exciton along the SWCNT is simulated by diffusion in a chosen time step. A class was written to handle saving and loading a calculation as well as easier handling of several methods for different scenarios of the interaction between the fluorescence decay and the dopamine. The underlying model for the simulation is flexible. In each simulation, the decay path of a set number 
of photons $n \_$photons is simulated to calculate the quantum yield of the excited state and the defect state. The length of the SWCNT CNT_length and the number of defects $n \_$defects are set at the beginning of the calculation, by distributing the defect sites randomly on the SWCNT. The exciton is initiated at a random position on the SWCNT in a mobile state, either the bright or the dark state. If the distance between two defects is less than the exciton radius $r_{-} e x c$, non-radiative quenching takes place at that position if the exciton is trapped there to account for the total quenching of SWCNTs with a large defect density. If the exciton is in one of the mobile states it defuses along the SWCNT in the timestep $t \_$step. For the exciton diffusion, the SWCNT is treated one dimensionally, since the exciton radius is large enough ${ }^{70}$ that it will likely hit the defect site when diffusing along the tube length. If the exciton reaches the end of the SWCNT, non-radiative quenching takes place. The exciton is trapped in the first defect site encountered along the diffusion path. At the end of each time step the probability for all depopulating processes of the excitons current state is calculated. The exciton converts to either another states or decays, upon which a new SWCNT with different random defect distribution is initiated and the process repeated to obtain the photo luminescence quantum yield. For the software, Python $3^{71}$ with the packages Numpy $(1.17)^{72}$, Matplotlib (1.3) ${ }^{73}$, Scipy $(1.3)^{74}$, H5py (2.9) $)^{75}$ and Ipykernel (5.1) were used.

\section{ASSOCIATED CONTENT}

\section{Corresponding Author}

* sebastian.kruss@rub.de 


\section{Funding Sources}

Volkswagen Foundation, Deutsche Forschungsgemeinschaft (DFG)

Notes

There are no competing financial interests.

SUPPORTING INFORMATION

Supporting Information is available.

\section{ACKNOWLEDGMENT}

We thank Tobias Hertel and Jana Zaumseil for fruitful discussions on exciton diffusion. This project was funded by the Deutsche Forschungsgemeinschaft (DFG, German Research Foundation) under Germany's Excellence Strategy - EXC 2033 - 390677874 - RESOLV. S.K. thanks the DFG for funding within the Heisenberg program. This project was supported by the VW foundation. L. F. K. and J. L. acknowledge funding by the Deutsche Forschungsgemeinschaft (DFG, German Research Foundation) under Germany's Excellence Strategy within the Cluster of Excellence PhoenixD (- EXC 2122 - 390833453).

\section{ABBREVIATIONS}




\section{TOC GRAPHICS}

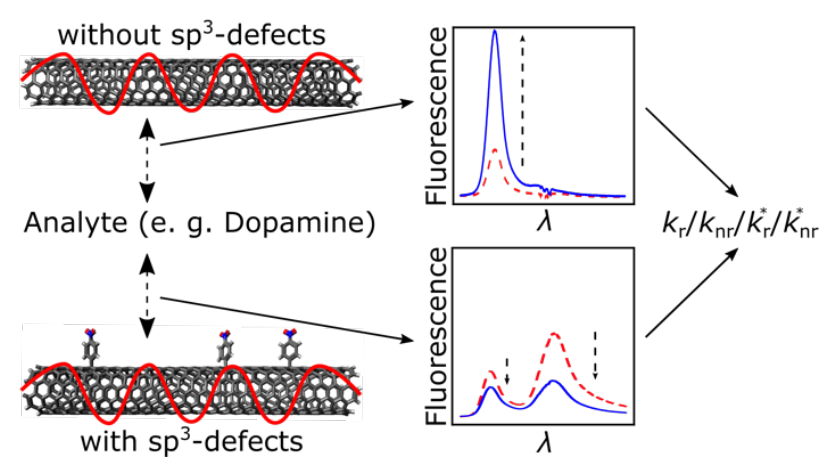




\section{REFERENCES}

(1) O’Connell, M. J.; Bachilo, S. M.; Huffman, C. B.; Moore, V. C.; Strano, M. S.; Haroz, E. H.; Rialon, K. L.; Boul, P. J.; Noon, W. H.; Kittrell, C.; Ma, J.; Hauge, R. H.; Weisman, R. B.; Smalley, R. E. Band Gap Fluorescence from Individual Single-Walled Carbon Nanotubes. Science 2002, 297 (5581), 593-596. https://doi.org/10.1126/science.1072631.

(2) Bachilo, S. M.; Strano, M. S.; Kittrell, C.; Hauge, R. H.; Smalley, R. E.; Weisman, R. B. Structure-Assigned Optical Spectra of Single-Walled Carbon Nanotubes. Science 2002, 298 (5602), 2361-2366. https://doi.org/10.1126/science.1078727.

(3) Kruss, S.; Hilmer, A. J.; Zhang, J.; Reuel, N. F.; Mu, B.; Strano, M. S. Carbon Nanotubes as Optical Biomedical Sensors. Adv. Drug Deliv. Rev. 2013, 65 (15), 1933-1950. https://doi.org/https://doi.org/10.1016/j.addr.2013.07.015.

(4) Hong, G.; Diao, S.; Antaris, A. L.; Dai, H. Carbon Nanomaterials for Biological Imaging and Nanomedicinal Therapy. Chem. Rev. 2015, 115 (19), 10816-10906. https://doi.org/10.1021/acs.chemrev.5b00008.

(5) Hendler-Neumark, A.; Bisker, G. Fluorescent Single-Walled Carbon Nanotubes for Protein Detection. Sensors . 2019. https://doi.org/10.3390/s19245403.

(6) Wang, F.; Dukovic, G.; Brus, L. E.; Heinz, T. F. The Optical Resonances in Carbon Nanotubes Arise from Excitons. Science 2005, 308 (5723), 838 LP - 841. https://doi.org/10.1126/science.1110265.

(7) Hagen, A.; Steiner, M.; Raschke, M. B.; Lienau, C.; Hertel, T.; Qian, H.; Meixner, A. J.; Hartschuh, A. Exponential Decay Lifetimes of Excitons in Individual Single-Walled Carbon Nanotubes. Phys. Rev. Lett. 2005, $95 \quad$ (19), 197401. https://doi.org/10.1103/PhysRevLett.95.197401. 
(8) Amori, A. R.; Hou, Z.; Krauss, T. D. Excitons in Single-Walled Carbon Nanotubes and Their Dynamics. Annu. Rev. Phys. Chem. 2018, 69 (1), 81-99. https://doi.org/10.1146/annurev-physchem-050317-014241.

(9) Hertel, T.; Himmelein, S.; Ackermann, T.; Stich, D.; Crochet, J. Diffusion Limited Photoluminescence Quantum Yields in 1-D Semiconductors: Single-Wall Carbon Nanotubes. ACS Nano 2010, 4 (12), 7161-7168. https://doi.org/10.1021/nn101612b.

(10) Zheng, M.; Jagota, A.; Semke, E. D.; Diner, B. A.; McLean, R. S.; Lustig, S. R.; Richardson, R. E.; Tassi, N. G. DNA-Assisted Dispersion and Separation of Carbon Nanotubes. Nat. Mater. 2003, 2 (5), 338-342. https://doi.org/10.1038/nmat877.

(11) Kam, N. W. S.; O\&\#039;Connell, M.; Wisdom, J. A.; Dai, H. Carbon Nanotubes as Multifunctional Biological Transporters and Near-Infrared Agents for Selective Cancer Cell Destruction. Proc. Natl. Acad. Sci. U. S. A. 2005, 102 (33), 11600 LP - 11605. https://doi.org/10.1073/pnas.0502680102.

(12) Kostarelos, K.; Bianco, A.; Prato, M. Promises, Facts and Challenges for Carbon Nanotubes in Imaging and Therapeutics. Nat. Nanotechnol. 2009, 4 (10), 627-633. https://doi.org/10.1038/nnano.2009.241.

(13) Gillen, A. J.; Kupis-Rozmysłowicz, J.; Gigli, C.; Schuergers, N.; Boghossian, A. A. Xeno Nucleic Acid Nanosensors for Enhanced Stability Against Ion-Induced Perturbations. J. Phys. Chem. Lett. 2018, 9 (15), 4336-4343. https://doi.org/10.1021/acs.jpclett.8b01879.

(14) Polo, E.; Nitka, T. T.; Neubert, E.; Erpenbeck, L.; Vuković, L.; Kruss, S. Control of Integrin Affinity by Confining RGD Peptides on Fluorescent Carbon Nanotubes. ACS Appl. Mater. Interfaces 2018, 10 (21), 17693-17703. https://doi.org/10.1021/acsami.8b04373.

(15) Wu, H.; Nißler, R.; Morris, V.; Herrmann, N.; Hu, P.; Jeon, S.-J.; Kruss, S.; Giraldo, J. P. 
Monitoring Plant Health with Near-Infrared Fluorescent H2O2 Nanosensors. Nano Lett. 2020, 20 (4), 2432-2442. https://doi.org/10.1021/acs.nanolett.9b05159.

(16) Prencipe, G.; Tabakman, S. M.; Welsher, K.; Liu, Z.; Goodwin, A. P.; Zhang, L.; Henry, J.; Dai, H. PEG Branched Polymer for Functionalization of Nanomaterials with Ultralong Blood Circulation. J. Am. Chem. Soc. 2009, 131 (13), 4783-4787. https://doi.org/10.1021/ja809086q.

(17) Bisker, G.; Dong, J.; Park, H. D. H. D.; Iverson, N. M. N. M.; Ahn, J.; Nelson, J. T. J. T.; Landry, M. P. M. P.; Kruss, S.; Strano, M. S. M. S. Protein-Targeted Corona Phase Molecular Recognition. Nat. Commun. 2016, 7, 1-14. https://doi.org/10.1038/ncomms10241.

(18) Mann, F. A.; Lv, Z.; Großhans, J.; Opazo, F.; Kruss, S.; Grosshans, J.; Opazo, F.; Kruss, S. Nanobody Conjugated Nanotubes for Targeted Near-Infrared in Vivo Imaging and Sensing. Angew. Chemie - Int. Ed. 2019, 11469-11473. https://doi.org/10.1002/anie.201904167.

(19) Antonucci, A.; Kupis-Rozmysłowicz, J.; Boghossian, A. A. Noncovalent Protein and Peptide Functionalization of Single-Walled Carbon Nanotubes for Biodelivery and Optical Sensing Applications. ACS Appl. Mater. Interfaces 2017, 9 (13), 11321-11331. https://doi.org/10.1021/acsami.7b00810.

(20) Harvey, J. D.; Baker, H. A.; Ortiz, M. V; Kentsis, A.; Heller, D. A. HIV Detection via a Carbon Nanotube RNA Sensor. ACS Sensors 2019, 4 (5), 1236-1244. https://doi.org/10.1021/acssensors.9b00025.

(21) Kim, J.-H.; Heller, D. A.; Jin, H.; Barone, P. W.; Song, C.; Zhang, J.; Trudel, L. J.; Wogan, G. N.; Tannenbaum, S. R.; Strano, M. S. The Rational Design of Nitric Oxide Selectivity in Single-Walled Carbon Nanotube near-Infrared Fluorescence Sensors for Biological 
Detection. Nat. Chem. 2009, 1 (6), 473-481. https://doi.org/10.1038/nchem.332.

(22) Safaee, M. M.; Gravely, M.; Roxbury, D. A Wearable Optical Microfibrous Biomaterial with Encapsulated Nanosensors Enables Wireless Monitoring of Oxidative Stress. Adv. $\begin{array}{lllll}\text { Funct } & \text { Mater. } & \text { 2021, } & 31 & \text { (13), }\end{array}$ https://doi.org/https://doi.org/10.1002/adfm.202006254.

(23) Nißler, R.; Bader, O.; Dohmen, M.; Walter, S. G.; Noll, C.; Selvaggio, G.; Groß, U.; Kruss, S. Remote near Infrared Identification of Pathogens with Multiplexed Nanosensors. Nat. Commun. 2020, 11 (1), 5995. https://doi.org/10.1038/s41467-020-19718-5.

(24) Reuel, N. F.; Grassbaugh, B.; Kruss, S.; Mundy, J. Z.; Opel, C.; Ogunniyi, A. O.; Egodage, K.; Wahl, R.; Helk, B.; Zhang, J.; Kalcioglu, Z. I.; Tvrdy, K.; Bellisario, D. O.; Mu, B.; Blake, S. S.; Van Vliet, K. J.; Love, J. C.; Wittrup, K. D.; Strano, M. S. Emergent Properties of Nanosensor Arrays: Applications for Monitoring IgG Affinity Distributions, Weakly Affined Hypermannosylation, and Colony Selection for Biomanufacturing. ACS Nano 2013, 7 (9), 7472-7482. https://doi.org/10.1021/nn403215e.

(25) Barone, P. W.; Strano, M. S. Single Walled Carbon Nanotubes as Reporters for the Optical Detection of Glucose. J. Diabetes Sci. Technol. 2009, 3 (2), 242-252. https://doi.org/10.1177/193229680900300204.

(26) Kruss, S.; Landry, M. P.; Vander Ende, E.; Lima, B. M. A.; Reuel, N. F.; Zhang, J.; Nelson, J.; Mu, B.; Hilmer, A.; Strano, M. Neurotransmitter Detection Using Corona Phase Molecular Recognition on Fluorescent Single-Walled Carbon Nanotube Sensors. J. Am. Chem. Soc. 2014, 136 (2), 713-724. https://doi.org/10.1021/ja410433b.

(27) Dinarvand, M.; Neubert, E.; Meyer, D.; Selvaggio, G.; Mann, F. A.; Erpenbeck, L.; Kruss, S. Near-Infrared Imaging of Serotonin Release from Cells with Fluorescent Nanosensors. 
Nano Lett. 2019, 19 (9), 6604-6611. https://doi.org/10.1021/acs.nanolett.9b02865.

(28) Bisker, G.; Bakh, N. A.; Lee, M. A.; Ahn, J.; Park, M.; O’Connell, E. B.; Iverson, N. M.; Strano, M. S. Insulin Detection Using a Corona Phase Molecular Recognition Site on Single-Walled Carbon Nanotubes. ACS Sensors 2018, 3 (2), 367-377. https://doi.org/10.1021/acssensors.7b00788.

(29) Jena, P. V; Roxbury, D.; Galassi, T. V; Akkari,L.; Horoszko, C. P.; Iaea, D. B.; Budhathokiuprety, J.; Pipalia, N.; Haka, A. S.; Harvey, J. D.; Mittal, J.; Max, F. R.; Joyce, J. A.; Heller, D. A. A Carbon Nanotube Optical Reporter Maps Endolysosomal Lipid Flux. ACS Nano 2017, 11 (11), 10689-10703. https://doi.org/10.1021/acsnano.7b04743.

(30) Mann, F. A.; Herrmann, N.; Meyer, D.; Kruss, S. Tuning Selectivity of Fluorescent Carbon Nanotube-Based Neurotransmitter Sensors. Sensors 2017, 17 (7), 1521. https://doi.org/10.3390/s17071521.

(31) Polo, E.; Kruss, S. Impact of Redox-Active Molecules on the Fluorescence of PolymerWrapped Carbon Nanotubes. J. Phys. Chem. C 2016, 120 (5). https://doi.org/10.1021/acs.jpcc.5b12183.

(32) Lee, A. J.; Wang, X.; Carlson, L. J.; Smyder, J. A.; Loesch, B.; Tu, X.; Zheng, M.; Krauss, T. D. Bright Fluorescence from Individual Single-Walled Carbon Nanotubes. Nano Lett. 2011, 11 (4), 1636-1640. https://doi.org/10.1021/n1200077t.

(33) Kruss, S.; Salem, D. P.; Vuković, L.; Lima, B.; Vander Ende, E.; Boyden, E. S.; Strano, M. S. High-Resolution Imaging of Cellular Dopamine Efflux Using a Fluorescent Nanosensor Array. Proc. Natl. Acad. Sci. 2017, 114 (8), 1789-1794. https://doi.org/10.1073/pnas.1613541114.

(34) Nißler, R.; Mann, F. A.; Chaturvedi, P.; Horlebein, J.; Meyer, D.; Vuković, L.; Kruss, S. 
Quantification of the Number of Adsorbed DNA Molecules on Single-Walled Carbon Nanotubes. J. Phys. Chem. C 2019, $123 \quad$ (8), 4837-4847. https://doi.org/10.1021/acs.jpcc.8b11058.

(35) Zhang, J.; Landry, M. P.; Barone, P. W.; Kim, J.-H.; Lin, S.; Ulissi, Z. W.; Lin, D.; Mu, B.; Boghossian, A. A.; Hilmer, A. J.; Rwei, A.; Hinckley, A. C.; Kruss, S.; Shandell, M. A.; Nair, N.; Blake, S.; Şen, F.; Şen, S.; Croy, R. G.; Li, D.; Yum, K.; Ahn, J.-H.; Jin, H.; Heller, D. A.; Essigmann, J. M.; Blankschtein, D.; Strano, M. S. Molecular Recognition Using Corona Phase Complexes Made of Synthetic Polymers Adsorbed on Carbon Nanotubes. Nat. Nanotechnol. 2013, 8 (12), 959-968. https://doi.org/10.1038/nnano.2013.236.

Zhang, J.; Boghossian, A. A.; Barone, P. W.; Rwei, A.; Kim, J.-H.; Lin, D.; Heller, D. A.; Hilmer, A. J.; Nair, N.; Reuel, N. F.; Strano, M. S. Single Molecule Detection of Nitric Oxide Enabled by d(AT)15 DNA Adsorbed to Near Infrared Fluorescent Single-Walled Carbon Nanotubes. J. Am. Chem. Soc. 2011, 133 (3), 567-581. https://doi.org/10.1021/ja1084942.

(37) Gravely, M.; Safaee, M. M.; Roxbury, D. Biomolecular Functionalization of a Nanomaterial To Control Stability and Retention within Live Cells. Nano Lett. 2019, 19 (9), 6203-6212. https://doi.org/10.1021/acs.nanolett.9b02267.

(38) Srivastava, A.; Htoon, H.; Klimov, V. I.; Kono, J. Direct Observation of Dark Excitons in Individual Carbon Nanotubes: Inhomogeneity in the Exchange Splitting. Phys. Rev. Lett. 2008, 101 (8), 87402. https://doi.org/10.1103/PhysRevLett.101.087402.

(39) Harutyunyan, H.; Gokus, T.; Green, A. A.; Hersam, M. C.; Allegrini, M.; Hartschuh, A. Defect-Induced Photoluminescence from Dark Excitonic States in Individual Single-Walled Carbon Nanotubes. Nano Lett. 2009, 9 (5), 2010-2014. https://doi.org/10.1021/n19002798. 
(40) Ju, S.-Y.; Kopcha, W. P.; Papadimitrakopoulos, F. Brightly Fluorescent Single-Walled Carbon Nanotubes via an Oxygen-Excluding Surfactant Organization. Science 2009, 323 (5919), 1319 LP - 1323. https://doi.org/10.1126/science.1166265.

(41) Cognet, L.; Tsyboulski, D. A.; Rocha, J.-D. R.; Doyle, C. D.; Tour, J. M.; Weisman, R. B. Stepwise Quenching of Exciton Fluorescence in Carbon Nanotubes by Single-Molecule Reactions. $\quad$ Science 2007, $316 \quad$ (5830), $1465 \quad$ LP $\quad-\quad 1468$. https://doi.org/10.1126/science.1141316.

(42) Ghosh, S.; Bachilo, S. M.; Simonette, R. A.; Beckingham, K. M.; Weisman, R. B. Oxygen Doping Modifies Near-Infrared Band Gaps in Fluorescent Single-Walled Carbon Nanotubes. $\quad$ Science $\quad 2010, \quad 330 \quad$ (6011), https://doi.org/10.1126/science.1196382.

(43) Miyauchi, Y.; Iwamura, M.; Mouri, S.; Kawazoe, T.; Ohtsu, M.; Matsuda, K. Brightening of Excitons in Carbon Nanotubes on Dimensionality Modification. Nat. Photonics 2013, 7 (9), 715-719. https://doi.org/10.1038/nphoton.2013.179.

(44) Piao, Y.; Meany, B.; Powell, L. R.; Valley, N.; Kwon, H.; Schatz, G. C.; Wang, Y. Brightening of Carbon Nanotube Photoluminescence through the Incorporation of Sp3 Defects. Nat. Chem. 2013, 5 (10), 840-845. https://doi.org/10.1038/nchem.1711.

(45) Ma, X.; Hartmann, N. F.; Baldwin, J. K. S.; Doorn, S. K.; Htoon, H. Room-Temperature Single-Photon Generation from Solitary Dopants of Carbon Nanotubes. Nat. Nanotechnol. 2015, 10 (8), 671-675. https://doi.org/10.1038/nnano.2015.136.

(46) Mann, F. A.; Herrmann, N.; Opazo, F.; Kruss, S. Quantum Defects as a Toolbox for the Covalent Functionalization of Carbon Nanotubes with Peptides and Proteins. Angew. Chemie - Int. Ed. 2020, 2-9. https://doi.org/10.1002/anie.202003825. 
(47) Janas, D. Perfectly Imperfect: A Review of Chemical Tools for Exciton Engineering in Single-Walled Carbon Nanotubes. Mater. Horizons 2020, 7 (11), 2860-2881. https://doi.org/10.1039/D0MH00845A.

(48) Nißler, R.; Kurth, L.; Li, H.; Spreinat, A.; Kuhlemann, I.; Flavel, B. S.; Kruss, S. Sensing with Chirality-Pure Near-Infrared Fluorescent Carbon Nanotubes. Anal. Chem. 2021, 93 (16), 6446-6455. https://doi.org/10.1021/acs.analchem.1c00168.

(49) Li, H.; Gordeev, G.; Garrity, O.; Reich, S.; Flavel, B. S. Separation of Small-Diameter Single-Walled Carbon Nanotubes in One to Three Steps with Aqueous Two-Phase Extraction. ACS Nano 2019, 13 (2), 2567-2578. https://doi.org/10.1021/acsnano.8b09579.

(50) Ishii, A.; Machiya, H.; Kato, Y. K. High Efficiency Dark-to-Bright Exciton Conversion in Carbon Nanotubes. Phys. Rev. $\quad X \quad$ 2019, 9 (4), 41048. https://doi.org/10.1103/PhysRevX.9.041048.

(51) He, X.; Velizhanin, K. A.; Bullard, G.; Bai, Y.; Olivier, J.-H.; Hartmann, N. F.; Gifford, B. J.; Kilina, S.; Tretiak, S.; Htoon, H.; Therien, M. J.; Doorn, S. K. Solvent- and WavelengthDependent Photoluminescence Relaxation Dynamics of Carbon Nanotube Sp ${ }^{3}$ Defect States. ACS Nano 2018, acsnano.8b02909. https://doi.org/10.1021/acsnano.8b02909.

(52) Kim, Y.; Velizhanin, K. A.; He, X.; Sarpkaya, I.; Yomogida, Y.; Tanaka, T.; Kataura, H.; Doorn, S. K.; Htoon, H. Photoluminescence Intensity Fluctuations and TemperatureDependent Decay Dynamics of Individual Carbon Nanotube Sp3 Defects. J. Phys. Chem. Lett. 2019, 10 (6), 1423-1430. https://doi.org/10.1021/acs.jpclett.8b03732.

(53) Schöppler, F.; Mann, C.; Hain, T. C.; Neubauer, F. M.; Privitera, G.; Bonaccorso, F.; Chu, D.; Ferrari, A. C.; Hertel, T. Molar Extinction Coefficient of Single-Wall Carbon Nanotubes. J. Phys. Chem. C 2011, 115 (30), 14682-14686. 
https://doi.org/10.1021/jp205289h.

(54) Perebeinos, V.; Avouris, P. Phonon and Electronic Nonradiative Decay Mechanisms of Excitons in Carbon Nanotubes. Phys. Rev. Lett. 2008, 101 (5), 57401. https://doi.org/10.1103/PhysRevLett.101.057401.

(55) Hartmann, N. F.; Velizhanin, K. A.; Haroz, E. H.; Kim, M.; Ma, X.; Wang, Y.; Htoon, H.; Doorn, S. K. Photoluminescence Dynamics of Aryl Sp3 Defect States in Single-Walled Carbon Nanotubes. ACS Nano 2016, 10 (9), 8355-8365.

(56) Arnold, M. S.; Blackburn, J. L.; Crochet, J. J.; Doorn, S. K.; Duque, J. G.; Mohite, A.; Telg, H. Recent Developments in the Photophysics of Single-Walled Carbon Nanotubes for Their Use as Active and Passive Material Elements in Thin Film Photovoltaics. Phys. Chem. Chem. Phys. 2013, 15 (36), 14896-14918. https://doi.org/10.1039/C3CP52752B.

(57) Gifford, B. J.; Kilina, S.; Htoon, H.; Doorn, S. K.; Tretiak, S. Controlling Defect-State Photophysics in Covalently Functionalized Single-Walled Carbon Nanotubes. Acc. Chem. Res.2020, 53 (9), 1791-1801. https://doi.org/10.1021/acs.accounts.0c00210.

(58) Kawabe, R.; Takaki, H.; Ibi, T.; Maeda, Y.; Nakagawa, K.; Maki, H. Pure and Efficient Single-Photon Sources by Shortening and Functionalizing Air-Suspended Carbon Nanotubes. ACS Appl. Nano Mater. 2020, 3 (1), 682-690. https://doi.org/10.1021/acsanm.9b02209.

(59) Siitonen, A. J.; Tsyboulski, D. A.; Bachilo, S. M.; Weisman, R. B. Surfactant-Dependent Exciton Mobility in Single-Walled Carbon Nanotubes Studied by Single-Molecule Reactions. Nano Lett. 2010, 10 (5), 1595-1599. https://doi.org/10.1021/n19039845.

(60) Chio, L.; Pinals, R. L.; Murali, A.; Goh, N. S.; Landry, M. P. Covalent Surface Modification Effects on Single-Walled Carbon Nanotubes for Targeted Sensing and Optical Imaging. 
$A d v$

Funct.

Mater.

2020 ,

30

(17),

1910556.

https://doi.org/https://doi.org/10.1002/adfm.201910556.

(61) Budhathoki-Uprety, J.; Shah, J.; Korsen, J. A.; Wayne, A. E.; Galassi, T. V; Cohen, J. R.; Harvey, J. D.; Jena, P. V; Ramanathan, L. V; Jaimes, E. A.; Heller, D. A. Synthetic Molecular Recognition Nanosensor Paint for Microalbuminuria. Nat. Commun. 2019, 10 (1), 3605. https://doi.org/10.1038/s41467-019-11583-1.

(62) Elizarova, S.; Chouaib, A.; Shaib, A.; Mann, F.; Brose, N.; Kruss, S.; Daniel, J. A. A Fluorescent Nanosensor Paint Reveals the Heterogeneity of Dopamine Release from Neurons at Individual Release Sites. bioRxiv 2021, 2021.03.28.437019. https://doi.org/10.1101/2021.03.28.437019.

(63) Lew, T. T. S.; Koman, V. B.; Silmore, K. S.; Seo, J. S.; Gordiichuk, P.; Kwak, S.-Y.; Park, M.; Ang, M. C.-Y.; Khong, D. T.; Lee, M. A.; Chan-Park, M. B.; Chua, N.-H.; Strano, M. S. Real-Time Detection of Wound-Induced H2O2 Signalling Waves in Plants with Optical Nanosensors. Nat. Plants 2020, 6 (4), 404-415. https://doi.org/10.1038/s41477-020-06324.

(64) Giraldo, J. P.; Wu, H.; Newkirk, G. M.; Kruss, S. Nanobiotechnology Approaches for Engineering Smart Plant Sensors. Nat. Nanotechnol. 2019, 14 (6), 541-553. https://doi.org/10.1038/s41565-019-0470-6.

(65) Nečas, D.; Klapetek, P. Gwyddion: An Open-Source Software for SPM Data Analysis. Open Phys. 10 (1), 181-188. https://doi.org/https://doi.org/10.2478/s11534-011-0096-2.

(66) de Mello, J. C.; Wittmann, H. F.; Friend, R. H. An Improved Experimental Determination of External Photoluminescence Quantum Efficiency. Adv. Mater. 1997, 9 (3), 230-232. https://doi.org/10.1002/adma.19970090308. 
(67) Graf, A.; Zakharko, Y.; Schießl, S. P.; Backes, C.; Pfohl, M.; Flavel, B. S.; Zaumseil, J. Large Scale, Selective Dispersion of Long Single-Walled Carbon Nanotubes with High Photoluminescence Quantum Yield by Shear Force Mixing. Carbon N. Y. 2016, 105, 593599. https://doi.org/https://doi.org/10.1016/j.carbon.2016.05.002.

(68) Manteiga Vázquez, F.; Yu, Q.; Klepzig, L. F.; Siebbeles, L. D. A.; Crisp, R. W.; Lauth, J. Probing Excitons in Ultrathin PbS Nanoplatelets with Enhanced Near-Infrared Emission. $J$. Phys. Chem. Lett. 2021, 12 (1), 680-685. https://doi.org/10.1021/acs.jpclett.0c03461.

(69) Spoor, F. C. M.; Kunneman, L. T.; Evers, W. H.; Renaud, N.; Grozema, F. C.; Houtepen, A. J.; Siebbeles, L. D. A. Hole Cooling Is Much Faster than Electron Cooling in PbSe Quantum Dots. ACS Nano 2016, $10 \quad$ (1), 695-703. https://doi.org/10.1021/acsnano.5b05731.

(70) Lüer, L.; Hoseinkhani, S.; Polli, D.; Crochet, J.; Hertel, T.; Lanzani, G. Size and Mobility of Excitons in $(6,5)$ Carbon Nanotubes. Nat. Phys. 2009, 5 (1), 54-58. https://doi.org/10.1038/nphys1149.

(71) Van Rossum, G.; Drake, F. L. Python 3 Reference Manual; CreateSpace. Scotts Val. CA 2009.

(72) Harris, C. R.; Millman, K. J.; van der Walt, S. J.; Gommers, R.; Virtanen, P.; Cournapeau, D.; Wieser, E.; Taylor, J.; Berg, S.; Smith, N. J.; Kern, R.; Picus, M.; Hoyer, S.; van Kerkwijk, M. H.; Brett, M.; Haldane, A.; del Río, J. F.; Wiebe, M.; Peterson, P.; GérardMarchant, P.; Sheppard, K.; Reddy, T.; Weckesser, W.; Abbasi, H.; Gohlke, C.; Oliphant, T. E. Array Programming with NumPy. Nature 2020, 585 (7825), 357-362. https://doi.org/10.1038/s41586-020-2649-2.

(73) Hunter, J. D. Matplotlib: A 2D Graphics Environment. Comput. Sci. Eng. 2007, 9 (3), 90- 
95. https://doi.org/10.1109/MCSE.2007.55.

(74) Virtanen, P.; Gommers, R.; Oliphant, T. E.; Haberland, M.; Reddy, T.; Cournapeau, D.; Burovski, E.; Peterson, P.; Weckesser, W.; Bright, J.; van der Walt, S. J.; Brett, M.; Wilson, J.; Millman, K. J.; Mayorov, N.; Nelson, A. R. J.; Jones, E.; Kern, R.; Larson, E.; Carey, C. J.; Polat, İ.; Feng, Y.; Moore, E. W.; VanderPlas, J.; Laxalde, D.; Perktold, J.; Cimrman, R.; Henriksen, I.; Quintero, E. A.; Harris, C. R.; Archibald, A. M.; Ribeiro, A. H.; Pedregosa, F.; van Mulbregt, P.; Vijaykumar, A.; Bardelli, A. Pietro; Rothberg, A.; Hilboll, A.; Kloeckner, A.; Scopatz, A.; Lee, A.; Rokem, A.; Woods, C. N.; Fulton, C.; Masson, C.; Häggström, C.; Fitzgerald, C.; Nicholson, D. A.; Hagen, D. R.; Pasechnik, D. V; Olivetti, E.; Martin, E.; Wieser, E.; Silva, F.; Lenders, F.; Wilhelm, F.; Young, G.; Price, G. A.; Ingold, G.-L.; Allen, G. E.; Lee, G. R.; Audren, H.; Probst, I.; Dietrich, J. P.; Silterra, J.; Webber, J. T.; Slavič, J.; Nothman, J.; Buchner, J.; Kulick, J.; Schönberger, J. L.; de Miranda Cardoso, J. V.; Reimer, J.; Harrington, J.; Rodríguez, J. L. C.; Nunez-Iglesias, J.; Kuczynski, J.; Tritz, K.; Thoma, M.; Newville, M.; Kümmerer, M.; Bolingbroke, M.; Tartre, M.; Pak, M.; Smith, N. J.; Nowaczyk, N.; Shebanov, N.; Pavlyk, O.; Brodtkorb, P. A.; Lee, P.; McGibbon, R. T.; Feldbauer, R.; Lewis, S.; Tygier, S.; Sievert, S.; Vigna, S.; Peterson, S.; More, S.; Pudlik, T.; Oshima, T.; Pingel, T. J.; Robitaille, T. P.; Spura, T.; Jones, T. R.; Cera, T.; Leslie, T.; Zito, T.; Krauss, T.; Upadhyay, U.; Halchenko, Y. O.; Vázquez-Baeza, Y.; Contributors, S. 1. SciPy 1.0: Fundamental Algorithms for Scientific Computing in Python. Nat. Methods 2020, 17 (3), 261-272. https://doi.org/10.1038/s41592-019-0686-2.

Colette, A. Python and HDF5; O'Reilly, 2013. 